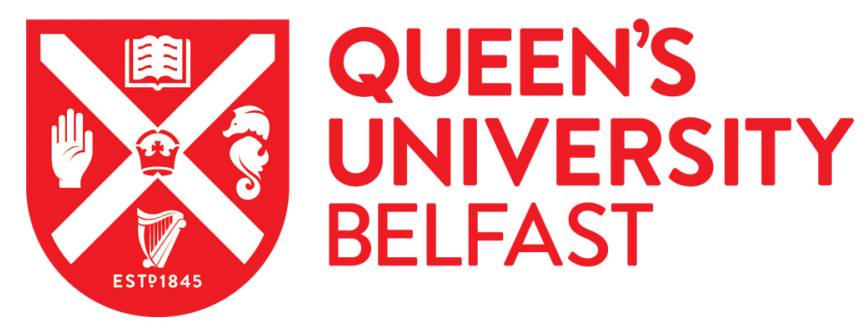

\title{
Two problems from the Polishchuk and Positselski book on Quadratic algebras
}

Shkarin, S., \& lyudu, N. (2018). Two problems from the Polishchuk and Positselski book on Quadratic algebras. Journal of Noncommutative Geometry, 12(1), 255-278. https://doi.org/10.4171/JNCG/276

Published in:

Journal of Noncommutative Geometry

Document Version:

Peer reviewed version

Queen's University Belfast - Research Portal:

Link to publication record in Queen's University Belfast Research Portal

Publisher rights

(c) 2018 EMS Publishing House. This work is made available online in accordance with the publisher's policies. Please refer to any applicable terms of use of the publisher.

\section{General rights}

Copyright for the publications made accessible via the Queen's University Belfast Research Portal is retained by the author(s) and / or other copyright owners and it is a condition of accessing these publications that users recognise and abide by the legal requirements associated with these rights.

Take down policy

The Research Portal is Queen's institutional repository that provides access to Queen's research output. Every effort has been made to ensure that content in the Research Portal does not infringe any person's rights, or applicable UK laws. If you discover content in the Research Portal that you believe breaches copyright or violates any law, please contact openaccess@qub.ac.uk. 


\title{
Two problems from the Polishchuk and Positselski book on Quadratic algebras
}

\author{
Natalia Iyudu and Stanislav Shkarin
}

January 17, 2018

\begin{abstract}
In the book 'Quadratic algebras' by Polishchuk and Positselski [23] algebras with a small number of generators $(n=2,3)$ are considered. For some number $r$ of relations possible Hilbert series are listed, and those appearing as series of Koszul algebras are specified. The first case, where it was not possible to do, namely the case of three generators $n=3$ and six relations $r=6$ is formulated as an open problem. We give here a complete answer to this question, namely for quadratic algebras with $\operatorname{dim} A_{1}=\operatorname{dim} A_{2}=3$, we list all possible Hilbert series, and find out which of them can come from Koszul algebras, and which can not.

As a consequence of this classification, we found an algebra, which serves as a counterexample to another problem from the same book 23. (Chapter 7, Sec. 1, Conjecture 2), saying that Koszul algebra of finite global homological dimension $d$ has $\operatorname{dim} A_{1} \geqslant d$. Namely, the 3-generated algebra $A$ given by relations $x x+y x=x z=z y=0$ is Koszul and its Koszul dual algebra $A^{!}$has Hilbert series of degree $4: H_{A^{!}}(t)=1+3 t+3 t^{2}+2 t^{3}+t^{4}$, hence $A$ has global homological dimension 4 .
\end{abstract}

MSC: $16 \mathrm{~A} 22,16 \mathrm{~S} 37,14 \mathrm{~A} 22$

Keywords: Quadratic algebras, Koszul algebras, Hilbert series, Gröbner basis

\section{Introduction}

Quadratic algebras have been studied intensely during the past several decades. Being interesting in their own right, they have many important applications in various parts of mathematics and physics including algebraic geometry, algebraic topology and group theory as well as in mathematical physics.

They frequently originate in physics. One example is that the 3-dimensional Sklyanin algebras were introduced and used in order to integrate a wide class of quantum systems on a lattice. These algebras have their Koszul duals in the class of quadratic algebras with $\operatorname{dim} A_{1}=\operatorname{dim} A_{2}=3$, the very class we study in this paper.

Quadratic algebras are noncommutative objects which lie in foundation of many noncommutative theories, for example, in work of A.Connes and M.Dubois-Violette [8], notions of noncommutative differential geometry obtain their purely algebraic counterpart through introducing the appropriate quadratic form on quadratic algebras. The big area of research generalizing notions of algebraic geometry to noncommutative spaces, due to Artin, Tate, Van den Bergh, Stafford etc. [3, 4, 5, 25] contains a great deal of studying structural and homological properties of quadratic algebras and their representations. Certain quadratic algebras serve as important examples for the notions of noncommutative (symplectic) spaces introduced by Kontsevich [18, 19], so information about general rules on the structure of such algebras makes it possible to describe examples explicitly.

We find it very important to study fundamental, most general properties of quadratic algebras, their Hilbert series, Koszulity, other homological properties, PBW type properties, etc. and to develop appropriate tools for that, which is a goal of present paper.

For further information on quadratic algebras, their Hilbert series and various aspects of their applications, we refer to $[1,2,17,14,15,17,16,123,4,21,24,13,26,22,10,11,6,120,27,12$ and references therein, however it will give still the list which is far from being exhaustive. 
Throughout this paper, $\mathbb{K}$ is an arbitrary field. For a $\mathbb{Z}_{+}$-graded vector space $B, B_{m}$ always stands for the $m^{\text {th }}$ component of $B$ and $H_{B}(t)=\sum_{j=0}^{\infty} \operatorname{dim} B_{j} t^{j}$ is the Hilbert series of $B$.

If $V$ is an $n$-dimensional vector space over $\mathbb{K}$, then $F=F(V)$ is the tensor algebra of $V$, which is naturally identified with the free algebra $\mathbb{K}\left\langle x_{1}, \ldots, x_{n}\right\rangle$ for any choice of a basis $x_{1}, \ldots, x_{n}$ in $V$. We often use the juxtaposition notation for the operation in $F(V)$ (for instance, we write $x_{j} x_{k}$ instead of $\left.x_{j} \otimes x_{k}\right)$. We always use the degree grading on $F$ : the $m^{\text {th }}$ graded component $F_{m}$ of $F$ is $V^{m}$. A degree graded algebra $A$ is a quotient of $F$ by a proper graded ideal $I$ ( $I$ is graded if it is the direct sum of $\left.I \cap F_{m}\right)$. This ideal is called the ideal of relations of $A$. If $x_{1}, \ldots, x_{n}$ is a fixed basis in $V$ and the monomials in $x_{j}$ carry an ordering compatible with the multiplication in $A$, we can speak of the Gröbner basis of the ideal of relations of $A$. If $\Lambda$ is the set of leading monomials of the elements of such a basis, then the normal words for $A$ are the monomials in $x_{j}$ featuring no element of $\Lambda$ as a submonomial. Normal words form a basis in $A$ as a $\mathbb{K}$-vector space. Thus, knowing the normal words implies knowing the Hilbert series.

If $R$ is a subspace of the $n^{2}$-dimensional space $V^{2}$, then the quotient of $F$ by the ideal $I(V, R)$ generated by $R$ is called a quadratic algebra and denoted $A(V, R)$. Following [23], we say that a quadratic algebra $A=A(V, R)$ is a $P B W$-algebra if there are linear bases $x_{1}, \ldots, x_{n}$ and $g_{1}, \ldots, g_{m}$ in $V$ and $R$ respectively such that $g_{1}, \ldots, g_{m}$ is a Gröbner basis of the ideal $I=I(V, R)$ with respect to some wellordering on the monomials in $x_{1}, \ldots, x_{n}$, compatible with multiplication. For a given basis $x_{1}, \ldots, x_{n}$ in $V$, we get a bilinear form on $\mathbb{K}\left\langle x_{1}, \ldots, x_{n}\right\rangle$ by setting $[u, v]=\delta_{u, v}$ for every pair of monomials $u$ and $v$ in $x_{1}, \ldots, x_{n}$. The quadratic algebra $A^{!}=A\left(V, R^{\perp}\right)$, where $R^{\perp}=\left\{u \in V^{2}:[r, u]=0\right.$ for each $\left.r \in R\right\}$, is called the Koszul dual algebra of $A$, we will call it also just dual algebra. Note that up to an isomorphism, the graded algebra $A^{!}$does not depend on the choice of a basis in $V$. It is well-known that $A$ is $\mathrm{PBW}$ if and only if $A^{!}$is PBW.

A degree graded algebra $A$ is called Koszul if the graded left $A$-module $\mathbb{K}$ (the structure is provided by the augmentation map) has a free resolution $\cdots \rightarrow M_{m} \rightarrow \cdots \rightarrow M_{1} \rightarrow A \rightarrow \mathbb{K} \rightarrow 0$ with the second last arrow being the augmentation map, and with each $M_{k}$ generated in degree $k$. The latter means that the matrices of the maps $M_{m} \rightarrow M_{m-1}$ with respect to some free bases consist of homogeneous elements of degree 1. Replacing left modules by the right ones leads to the same class of algebras. We use the following well-known properties of Koszul algebras:

every Koszul algebra is quadratic; every PBW-algebra is Koszul;

$A$ is Koszul $\Longleftrightarrow A^{!}$is Koszul;

$H_{A}(-t) H_{A^{!}}(t)=1$ if $A$ is Koszul.

In [23, Chapter 6 , Section 5] possible Hilbert series of Koszul algebras $A$ with small values of $\operatorname{dim} A_{1}$ and $\operatorname{dim} A_{2}$ are listed. The first case not covered there is $\operatorname{dim} A_{1}=\operatorname{dim} A_{2}=3$. In this case, only the Hilbert series of PBW algebras are given. It is stated in [23] that the complete list of Hilbert series of quadratic algebras satisfying $\operatorname{dim} A_{1}=\operatorname{dim} A_{2}=3$ as well as the complete list of the Hilbert series of Koszul algebras in this case are unknown. We fill this gap by proving the following results.

Theorem 1.1. For quadratic algebras $A$ satisfying $\operatorname{dim} A_{1}=\operatorname{dim} A_{2}=3$, the complete list of possible Hilbert series is $\left\{H_{1}, \ldots, H_{11}\right\}$, where

$$
\begin{array}{rlrl}
H_{1}(t) & =1+3 t+3 t^{2} ; & & H_{2}(t)=1+3 t+3 t^{2}+t^{3}=(1+t)^{3} \\
H_{3}(t)=1+3 t+3 t^{2}+t^{3}+t^{4}+t^{5}+\cdots=\frac{1+2 t-2 t^{3}}{1-t} ; & H_{4}(t)=1+3 t+3 t^{2}+2 t^{3} ; \\
H_{5}(t)=1+3 t+3 t^{2}+2 t^{3}+t^{4} ; & H_{6}(t)=1+3 t+3 t^{2}+2 t^{3}+t^{4}+t^{5}+\cdots=\frac{1+2 t-t^{3}-t^{4}}{1-t} ; \\
H_{7}(t)=1+3 t+3 t^{2}+2 t^{3}+2 t^{4}+2 t^{5}+\cdots=\frac{1+2 t-t^{3}}{1-t} ; & H_{8}(t)=1+3 t+3 t^{2}+3 t^{3}+3 t^{4}+3 t^{5}+\cdots=\frac{1+2 t}{1-t} ; \\
H_{9}(t)=1+3 t+3 t^{2}+4 t^{3}+4 t^{4}+4 t^{5}+\cdots=\frac{1+2 t+t^{3}}{1-t} ; & H_{10}(t)=1+3 t+3 t^{2}+4 t^{3}+5 t^{4}+6 t^{5}+\cdots=\frac{1+t-2 t^{2}+t^{3}}{(1-t)^{2}} ; \\
H_{11}(t)=1+3 t+3 t^{2}+5 t^{3}+8 t^{4}+13 t^{5}+\cdots=\frac{1+2 t-t^{2}-t^{3}}{1-t-t^{2}}, &
\end{array}
$$

where the last series, starting from the third term, is formed by consecutive Fibonacci numbers.

As we have mentioned above, the complete list of Hilbert series of PBW algebras $A$ satisfying $\operatorname{dim} A_{1}=\operatorname{dim} A_{2}=3$ can be found in [23. It consists of $H_{j}$ with $j \in\{2,7,8,9,10,11\}$. 
Theorem 1.2. For Koszul algebras $A$ satisfying $\operatorname{dim} A_{1}=\operatorname{dim} A_{2}=3$, the complete list of Hilbert series consists of $H_{j}$ with $j \in\{2,5,6,7,8,9,10,11\}$. That is, for each $j \in\{2,5,6,7,8,9,10,11\}$, there is a Koszul algebra $A$ satisfying $H_{A}=H_{j}$, while for $j \in\{1,3,4\}$, every quadratic algebra $A$ satisfying $H_{A}=H_{j}$ is non-Koszul.

As a consequence of these classification Theorems 1.1 and 1.2, we can find an algebra, which serves as a counterexample to another problem formulated in the Polishchuk and Positselski book [23].

Conjecture 1.3. ([23], Chapter 7, Sec. 1, Conjecture 2) Any Koszul algebra of finite global homological dimension $d$ has $\operatorname{dim} A_{1} \geqslant d$. By duality this is equivalent to the following statement: for a Koszul algebra $B$ with $B_{d+1}=0$ and $B_{d} \neq 0$ one has $\operatorname{dim} B_{1} \geqslant d$.

We can consider algebra $A_{5}$ from the Table 1 . It is given by relations $x x-y x=x y=y y=y x=z x=$ $z z=0$. The Hilbert series of this algebra is $H_{A}(t)=H_{5}=1+3 t+3 t^{2}+2 t^{3}+t^{4}$, so it is a polynomial of degree 4. Then quite straightforward arguments in Proposition 4.2 ensure that the Koszul dual algebra $A_{5}^{!}$, given by relations $x x+y x=x z=z y=0$, is Koszul. This provides a counterexample to Conjecture 1.3. Namely, the algebra $A^{!}$serves as a counterexample to the first part of the conjecture: it has a global homological dimension 4 . The algebra $A$ itself is a counterexample to the second statement of the conjecture, since its Hilbert series is a polynomial of degree 4.

Note that the list of series acquires two extra members $H_{5}$ and $H_{6}$ when the PBW condition is relaxed to Koszulity.

The key lemma, allowing to manage all possibilities, is the following linear algebra statement.

Lemma 1.4. Let $V$ be a 3-dimensional vector space over an infinite field $\mathbb{K}$ and $R$ be a 6-dimensional subspace of $V \otimes V$. Then at least one of the following statements is true:

(P1) there is a 1-dimensional subspace $L \subset V$ such that $(V \otimes L) \oplus R=V \otimes V$ or $(L \otimes V) \oplus R=V \otimes V$;

(P2) there is 1-dimensional subspace $L \subset V$ such that $V \otimes L \subset R$ or $L \otimes V \subset R$;

(P3) there is an invertible linear map $T: V \rightarrow V$ such that $R=\operatorname{span}\{x \otimes T x: x \in V\}$.

While (P1) and (P2) are not mutually exclusive, (P3) is incompatible with each of (P1) and (P2).

In Section 2 we show that the Hilbert series of any quadratic algebra $A$, satisfying $\operatorname{dim} A_{1}=\operatorname{dim} A_{2}=$ 3 belongs to $\left\{H_{1}, \ldots, H_{11}\right\}$, applying Lemma 1.4 and Gröbner basis techniques. Then in Section 3 we give a proof of Lemma 1.4. In Section 4 we show that $H_{j}$ is the Hilbert series of a quadratic algebra $A$ for $1 \leqslant j \leqslant 11$. We also observe that $A$ can be chosen Koszul if $j \in\{2,5,6,7,8,9,10,11\}$ and that every algebra $A$ with $H_{A}=H_{j}$ for $j \in\{1,3,4\}$ is non-Koszul, thus completing the proofs of Theorems 1.1 and 1.2 .

Throughout the paper, when talking of Gröbner bases, assume that the monomials carry the leftto-right degree lexicographical ordering with the variables ordered by $x>y>z$ or $x_{1}>x_{2}>x_{3}$ (depending on how the variables are called in each case).

\section{Admissible series}

In this section we apply Lemma 1.4 and Gröbner basis arguments to prove the following result. Next section will be dedicated to the proof of the Lemma 1.4 itself.

Proposition 2.1. Let $A$ be a quadratic $\mathbb{K}$-algebra satisfying $\operatorname{dim} A_{1}=\operatorname{dim} A_{2}=3$. Then $H_{A} \in$ $\left\{H_{1}, \ldots, H_{11}\right\}$.

Since replacing the ground field $\mathbb{K}$ by a field extension does not change the Hilbert series of an algebra given by generators and relations, for the purpose of proving Proposition 2.1, we can without loss of generality assume that $\mathbb{K}$ is algebraically closed. Then $\mathbb{K}$ is infinite. By Lemma 1.4, Proposition 2.1 is an immediate corollary of the following three lemmas. We essentially consider three possibilities given by Lemma 1.4, and in each case find out which series are possible, looking mainly at the shape of the Gröbner basis. 
Lemma 2.2. Let $V$ be a 3-dimensional vector space over $\mathbb{K}$ and $R$ be a 6-dimensional subspace of $V \otimes V$ such that condition (P1) of Lemma 1.4 is satisfied. Then for the quadratic algebra $A=A(V, R)$, $H_{A} \in\left\{H_{1}, \ldots, H_{8}\right\}$.

Lemma 2.3. Let $V$ be a 3-dimensional vector space over an algebraically closed field $\mathbb{K}$ and $R$ be a 6-dimensional subspace of $V \otimes V$ such that condition (P2) of Lemma 1.4 is satisfied, while (P1) fails. Then for the quadratic algebra $A=A(V, R), H_{A} \in\left\{H_{1}, H_{2}, H_{3}, H_{7}, H_{9}, H_{10}, H_{11}\right\}$.

Lemma 2.4. Let $V$ be a 3-dimensional vector space over $\mathbb{K}$ and $R \subset V \otimes V$ be a subspace satisfying condition (P3) of Lemma 1.4. Then for the quadratic algebra $A=A(V, R), H_{A}=H_{2}$.

Proof of Lemma 2.2. Since (P1) is satisfied, there is a 1-dimensional subspace $L \subset V$ such that $(V \otimes$ $L) \oplus R=V \otimes V$ or $(L \otimes V) \oplus R=V \otimes V$. These two cases reduce to each other by passing to the algebra with the opposite multiplication. Thus we can assume that $(L \otimes V) \oplus R=V \otimes V$. Pick a basis $x_{1}, x_{2}, x_{3}$ in $V$ such that $x_{3}$ spans $L$. Since $(L \otimes V) \oplus R=V \otimes V$, there is a linear basis in $R$ of the form (we skip the symbol $\otimes$ for the rest of the proof):

$$
r_{j, k}=x_{j} x_{k}-x_{3} u_{j, k} \text { for } 1 \leqslant j \leqslant 2 \text { and } 1 \leqslant k \leqslant 3 \text {, where } u_{j, k} \in V .
$$

It follows that in the algebra $A, A_{2}=x_{3} V=x_{3} A_{1}$. Then $A_{3}=A_{2} V=x_{3} V V=x_{3} A_{2}=x_{3}^{2} V$. Iterating, we get $A_{n}=x_{3} A_{n-1}=x_{3}^{n-1} V$ for each $n \geqslant 2$. In particular, $\operatorname{dim} A_{n} \leqslant \operatorname{dim} A_{n-1} \leqslant 3$ for each $n \geqslant 2$. We also know that $\operatorname{dim} A_{1}=\operatorname{dim} A_{2}=3$.

Case 1: $\operatorname{dim} A_{3}=3$. This can only happen if $r_{j, k}$ form a Gröbner basis of the ideal $I=I(V, R)$. Since the leading monomials of these relations are $x_{j} x_{k}$ for $1 \leqslant j \leqslant 2$ and $1 \leqslant k \leqslant 3$, the normal words of degree $n \geqslant 3$ are $x_{3}^{n-1} x_{j}$ for $1 \leqslant j \leqslant 3$. Hence $\operatorname{dim} A_{n}=3$ for $n \geqslant 3$ and $H_{A}=H_{8}$.

Case 2: $\operatorname{dim} A_{3}=2$. This happens when there is exactly one degree 3 element $g$ of the Gröbner basis of $I$. The leading monomial of $g$ must have the shape $x_{3}^{2} x_{j}$ with $1 \leqslant j \leqslant 3$. If $j=3$, we have $g=x_{3}^{3}$ and $x_{3}^{3}=0$ in $A$. Hence for $n \geqslant 4, A_{n}=x_{3}^{n-1} V=\{0\}$. Thus $H_{A}=1+3 t+3 t^{2}+2 t^{3}=H_{4}$. It remains to consider the case $j \in\{1,2\}$. Swapping $x_{1}$ and $x_{2}$, if necessary, we can without loss of generality assume that $j=1$. We know that $\operatorname{dim} A_{4} \leqslant \operatorname{dim} A_{3}=2$. The case $\operatorname{dim} A_{4}=2$ can only happen if the relations $r_{j, k}$ together with $g$ form a Gröbner basis of $I$. In this case the normal words of degree $n \geqslant 3$ are $x_{3}^{n-1} x_{k}$ with $k \in\{2,3\}$. This gives $H_{A}=1+3 t+3 t^{2}+2 t^{3}+2 t^{4}+2 t^{5}+\cdots=H_{7}$. It remains to consider the case $\operatorname{dim} A_{4}=1$. This happens when there is exactly one degree 4 element $h$ in the Gröbner basis of $I$. The leading monomial of $h$ must have the shape $x_{3}^{3} x_{k}$ with $2 \leqslant k \leqslant 3$. If $k=3$, we have $h=x_{3}^{4}$ and $x_{3}^{4}=0$ in $A$. Hence for $n \geqslant 5, A_{n}=x_{3}^{n-1} V=\{0\}$. Thus $H_{A}=1+3 t+3 t^{2}+2 t^{3}+t^{4}=H_{5}$. Assume now that $k=2$. If the relations $r_{j, k}$ together with $g$ and $h$ do not form the Gröbner basis of $I$, there is a degree 5 element $q$ of this Gröbner basis. By looking at the leading terms of $r_{j, k}, g$ and $h$, we see that the only possibility is for $q$ to be equal $x_{3}^{5}$ up to a non-zero scalar multiple. Again, this gives $H_{A}=H_{5}$. On the other hand, if $r_{j, k}$ together with $g$ and $h$ do form the Gröbner basis of $I$, the only normal word of degree $n \geqslant 4$ is $x_{3}^{n}$. Hence $H_{A}=1+3 t+3 t^{2}+2 t^{3}+t^{4}+t^{5}+\cdots=H_{6}$.

Case 3: $\operatorname{dim} A_{3}=1$. This happens when there are exactly two degree 3 elements $g$ and $h$ of the Gröbner basis of $I$. By swapping $g$ and $h$, if necessary, we can assume that the leading terms of $g$ and $h$ are $x_{3}^{2} x_{j}$ and $x_{3}^{2} x_{k}$ respectively with $1 \leqslant j<k \leqslant 3$. If $k=3$, we have $h=x_{3}^{3}$ and $x_{3}^{3}=0$ in $A$. Hence for $n \geqslant 4, A_{n}=x_{3}^{n-1} V=\{0\}$. Thus $H_{A}=1+3 t+3 t^{2}+t^{3}=H_{2}$. It remains to consider the case $j=1, k=2$. If the relations $r_{j, k}$ together with $g$ and $h$ do not form the Gröbner basis of $I$, there is a degree 4 element $q$ in this Gröbner basis. By looking at the leading terms of $r_{j, k}, g$ and $h$, we see that the only possibility is for $q$ to be equal $x_{3}^{4}$ up to a non-zero scalar multiple. Again, this gives $H_{A}=H_{2}$. If $r_{j, k}$ together with $g$ and $h$ do form the Gröbner basis of $I$, the only normal word of degree $n \geqslant 3$ is $x_{3}^{n}$. Hence $H_{A}=1+3 t+3 t^{2}+t^{3}+t^{4}+t^{5}+\cdots=H_{3}$.

Case 4: $\operatorname{dim} A_{3}=0$. Obviously, $H_{A}=1+3 t+3 t^{2}=H_{1}$.

Proof of Lemma 2.3. Since (P2) holds, there is a 1-dimensional subspace $L$ of $V$ such that $V L \subset R$ or $L V \subset R$ (we skip the symbol $\otimes$ throughout the proof). The cases $V L \subset R$ and $L V \subset R$ reduce to each 
other by passing to the algebra with the opposite multiplication. Thus we can assume that $L V \subset R$. Pick $x$ in $V$, which spans $L$. Since (P1) fails,

$$
\text { for each } u \in V \backslash\{0\} \text {, there is } v=v(u) \in V \backslash\{0\} \text { such that } u v \in R \text {. }
$$

We shall verify that there are $y, z \in V$ such that $x, y, z$ is a basis in $V$ and at least one of the following conditions holds:

$$
\begin{aligned}
& R=\operatorname{span}\{x x, x y, x z, y x, z x, h\} \quad \text { with } h \in\{y y, y z-a z y, y z-z y+z z\}(a \in \mathbb{K}) \\
& R=\operatorname{span}\{x x, x y, x z, y y, z y, h\} \quad \text { with } h \in\{y x-z z, y z-z x, y x, y z, z x, z z\} \\
& R=\operatorname{span}\{x x, x y, x z, y y, z z, h\} \quad \text { with } h \in\{y x+z x, y z+z y, y x+z x-y z-z y\}
\end{aligned}
$$

Case 1: $V L \subset R$. Pick arbitrary $u, v \in V$ such that $x, u, v$ is a basis in $V$. Then the 5-dimensional space $L V+V L$ spanned by $S_{0}=\{x x, x u, x v, u x, v x\}$ is contained in $R$. Since $R$ is 6 -dimensional, it is spanned by $S_{0} \cup\{f\}$, where $f=a u u+b u v+c v u+d v v$ with $(a, b, c, d) \in \mathbb{K}^{4},(a, b, c, d) \neq(0,0,0,0)$. Since $\mathbb{K}$ is algebraically closed, there is a non-zero $(p, s) \in \mathbb{K}^{2}$ such that $a p^{2}+(b+c) p s+d s^{2}=0$. Next, pick $(q, t) \in \mathbb{K}^{2}$ such that $(p, s)$ and $(q, t)$ are linearly independent. The non-degenerate linear substitution, in which old $u$ and $v$ are replaced by $p u+q v$ and $s u+t v$ respectively, transforms $f$ into $g=\alpha u v+\beta v u+\gamma v v$ with non-zero $(\alpha, \beta, \gamma) \in \mathbb{K}^{3}$. If $\alpha=0$ and $\beta \neq 0$, we set $y=v$ and $z=\beta u+\gamma v$, while if $\alpha \neq 0$ and $\beta=0$, we set $y=\alpha u+\gamma v$ and $z=u$. This substitution transforms $g$ into a (non-zero) scalar multiple of $y z$. Now, with respect to the basis $x, y, z, R$ is spanned by $S \cup\{y z\}$ with $S=\{x x, x y, x z, y x, z x\}$. If $\alpha=\beta=0$, we set $y=v$ and $z=u$ and observe that $R$ is spanned by $S \cup\{y y\}$. If $\alpha \beta \neq 0$ and $\alpha+\beta \neq 0$, we set $y=u+\frac{\gamma v}{\alpha+\beta}, z=v$ and observe that $R$ is spanned by $S \cup\left\{y z+\frac{\beta}{\alpha} z y\right\}$. If $\alpha \beta \neq 0$ and $\alpha+\beta=\gamma=0$, with respect to $y=u$ and $z=v, R$ is spanned by $S \cup\{y z-z y\}$. Finally, if $\alpha \beta \gamma \neq 0$ and $\alpha+\beta=0$, we set $y=\frac{\alpha u}{\gamma}$ and $z=v$, with respect to which $R$ is spanned by $S \cup\{y z-z y+z z\}$. Thus (2.3) is satisfied provided $V L \subset R$.

Case 2: $V M \subset R$ for a 1-dimensional subspace $M$ of $V$ such that $M \neq L$. Pick $u \in M \backslash\{0\}$. Since $L \neq M, x$ and $u$ are linearly independent. For $w \in V$ such that $x, u, w$ is a basis in $V, R=$ $\operatorname{span}\left(S_{0} \cup\{f\}\right)$, where $S_{0}=\{x x, x u, x w, u u, w u\}$ and $f=a u x+b u w+c w x+d w w$ with $(a, b, c, d) \in \mathbb{K}^{4}$, $(a, b, c, d) \neq(0,0,0,0)$. For $\alpha \in \mathbb{K}^{*}$ and $p, q \in \mathbb{K}$ we can consider the basis $x, y, z$ in $V$ defined by $u=\alpha y$ and $w=z+p x+q y$. A direct computation shows that with respect to this basis, $R=\operatorname{span}(S \cup\{g\})$, where $S=\{x x, x y, x z, y y, z y\}$ and

$$
g=(a \alpha+b p \alpha+c q+d p q) y x+(b \alpha+d q) y z+(c+d p) z x+d z z .
$$

If $d \neq 0$ and $a d=b c$, by choosing $\alpha=1, q=-\frac{b}{d}$ and $p=-\frac{c}{d}$, we turn $g$ into a (non-zero) scalar multiple of $z z$. If $d=0$ and $a d \neq b c$, by choosing $\alpha=\frac{d^{2}}{b c-a d}, q=-\frac{b}{d}$ and $p=-\frac{c}{d}$, we turn $g$ into a scalar multiple of $y x-z z$. If $d=0$ and $b c \neq 0$, by choosing $\alpha=-\frac{c}{b}, p=0$ and $q=\frac{a}{b}$, we turn $g$ into a scalar multiple of $y z-z x$. If $b=d=0$ and $c \neq 0$, by choosing $\alpha=1, p=0$ and $q=-\frac{a}{c}$, we turn $g$ into a scalar multiple of $z x$. If $c=d=0$ and $b \neq 0$, by choosing $\alpha=1, q=0$ and $p=-\frac{a}{b}$, we turn $g$ into a scalar multiple of $y z$. Finally, if $b=c=d=0$, by choosing $\alpha=1$ and $p=q=0$, we turn $g$ into a scalar multiple of $y x$. Thus (2.4) is satisfied provided $V M \subset R$ for a 1-dimensional subspace $M$ different from $L$.

Case 3: $\quad V M \nsubseteq R$ for every 1-dimensional subspace $M$ of $V$. This is precisely the negation of the assumptions of Cases 1 and 2 . First, we shall verify that in this case

$$
y z \notin R \text { whenever } x, y, z \text { is a basis in } V \text {. }
$$

We argue by contradiction. Assume that (2.6) fails. Then there are $y, z \in V$ such that $x, y, z$ is a basis in $V$ and $y z \in R$. By (2.2), there are non-zero $(a, b, c),(p, q, r) \in \mathbb{K}^{3}$ such that $z(a x+b y+c z),(y+z)(p x+$ $q y+r z) \in R$. The assumption of Case 3 implies linear independence of $z, a x+b y+c z$ and $p x+q y+r z$. Indeed, if they were linearly dependent, using the inclusions $y z, z(a x+b y+c z),(y+z)(p x+q y+r z) \in R$, one easily finds a non-zero $u \in V$ such that $y u, z u \in R$. Since $x u \in R$, this implies $V M \subset R$ with $M$ 
being the linear span of $u$. Linear independence of $z, a x+b y+c z$ and $p x+q y+r z$ implies that $a q \neq b p$ and that

$$
R=\operatorname{span}\{x x, x y, x z, y z, z(a x+b y+c z),(y+z)(p x+q y+r z)\} .
$$

Since $\mathbb{K}$ is infinite, we can pick $\theta \in \mathbb{K} \backslash\{0,1\}$. By (2.2), there is a non-zero $(\alpha, \beta, \gamma) \in \mathbb{K}^{3}$ such that $(y+\theta z)(\alpha x+\beta y+\gamma z) \in R$. By (2.7), there exist $c_{1}, c_{2}, c_{3} \in \mathbb{K}$ such that

$$
(y+\theta z)(\alpha x+\beta y+\gamma z)=c_{1} y z+c_{2} z(a x+b y+c z)+c_{3}(y+z)(p x+q y+r z),
$$

where the equality holds in $\mathbb{K}\langle x, y, z\rangle$. Opening up the brackets in the above display, we obtain

$$
\alpha-p c_{3}=\beta-q c_{3}=\gamma-c_{1}-r c_{3}=\alpha \theta-a c_{2}-q c_{3}=\beta \theta-b c_{2}-q c_{3}=\gamma \theta-c c_{2}-r c_{3}=0 .
$$

Plugging $\alpha=p c_{3}$ and $\beta=q c_{3}$ into $\alpha \theta-a c_{2}-q c_{3}=\beta \theta-b c_{2}-q c_{3}=0$, we get $b c_{2}+(1-\theta) q c_{3}=$ $a c_{2}+(1-\theta) p c_{3}=0$. Since $\theta \neq 1$ and $a q \neq b p$, the determinant $(1-\theta)(b p-a q)$ of this system of two linear equations on $c_{2}$ and $c_{3}$ is non-zero. Hence $c_{2}=c_{3}=0$. Since $\theta \neq 0$, the above display implies $\alpha=p c_{3}=0, \beta=q c_{3}=0$ and $\gamma=\frac{c c_{2}+r c_{3}}{\theta}=0$, which contradicts $(\alpha, \beta, \gamma) \neq(0,0,0)$. This contradiction proves (2.6) .

Now (2.6) together with (2.2) imply that

$$
\text { for each } u \in V \backslash L \text {, there is }\left(a_{u}, b_{u}\right) \in \mathbb{K}^{2} \backslash\{(0,0)\} \text { such that } u\left(a_{u} u+b_{u} x\right) \in R \text {. }
$$

Observe that $a_{u} \neq 0$ for a Zarisski generic $u \in V$. Indeed, otherwise $V L \subset R$. Next, $b_{u} \neq 0$ for a Zarisski generic $u \in V$. Indeed, otherwise $R$ contains the 6 -dimensional space $\mathcal{S}$ of symmetric elements of $V^{2}$. Since $R$ also contains $L V$ and $L V \cap \mathcal{S}$ is one-dimensional, $\operatorname{dim} R \geqslant 8>6$, which is a contradiction. Thus we can pick $s, t \in V$ such that $x, s, t$ is a basis in $V$ and $a_{s} b_{s} a_{t} b_{t} \neq 0$. Now, using the inclusions $s\left(a_{s} s+b_{s} s\right), t\left(a_{t} t+b_{t} x\right) \in \mathbb{R}$, we can pick $p, q \in \mathbb{K}^{*}$ such that for $u=p s$ and $v=q t$, $u(x-u), v(x-v) \in R$. For $a=a_{u+v}$ and $b=b_{u+v}$, according to (2.8), we have $(a, b) \neq(0,0)$ and $(u+v)(a u+a v+b x) \in R$. Then $R=\operatorname{span}\{x x, x u, x v, u(x-u), v(x-v),(u+v)(a u+a v+b x)\}$. Now set $y=x-u$ and $z=x-v$. With respect to the basis $x, y, z$, the last equality can be rewritten as $R=\operatorname{span}(S \cup\{c(y+z) x-a(y z+z y)\}$, where $S=\{x x, x y, x z, y y, z z\}$ with $c=b+2 a$. If $c=0$, then $R=\operatorname{span}(S \cup\{y z+z y\})$. If $a=0$, then $R=\operatorname{span}(S \cup\{y x+z x\})$. If $a c \neq 0$, then replacing $y$ and $z$ by $\alpha y$ and $\alpha z$ for an appropriate $\alpha \in \mathbb{K}^{*}$, we get $R=\operatorname{span}(S \cup\{y x+z x-y z-z y\})$. Thus (2.5) is satisfied provided $V M \nsubseteq R$ for every 1-dimensional subspace $M$ of $V$.

It remains to determine the Hilbert series of $A=A(V, R)$ when $R$ satisfies one of the conditions (2.3), (2.4) or (2.5). If (2.3) is satisfied, the defining relations $x x, x y, x z, y x, z x$, and $h$ of $A$ form a Gröbner basis of the ideal $I=I(V, R)$. If $h=y z-a z y$ or $h=y z-z y+z z$, then the normal words of degree $n \geqslant 2$ are $z^{k} y^{n-k}$ for $0 \leqslant k \leqslant n$. Since there are $n+1$ of them, we have $H_{A}=H_{10}$. If $h=y y$, then the normal words of degree $n \geqslant 2$ are all monomials in $y$ and $z$, which do not contain $y y$ as a submonomial. It is easy to see that the number $a_{n}$ of such monomials satisfies the recurrent relation $a_{n+2}=a_{n+1}+a_{n}$, which together with $a_{2}=3$ and $a_{3}=5$ implies $H_{A}=H_{11}$.

Next, assume that (2.4) is satisfied. That is, $A$ is given by the relations $x x, x y, x z, y y, z y$ and $h$ with $h \in\{y x-z z, y z-z x, y x, y z, z x, z z\}$. If $h=y x-z z$, the defining relations together with $y z z$, $z z x, z z y$ and $z z z$ form a Gröbner basis of $I$. The only normal word of degree $\geqslant 3$ is $y z x$, which gives $H_{A}=H_{2}$. If $h=y z-z x$, then the defining relations together with $z z x$ form a Gröbner basis of $I$. The only normal word of degree $n \geqslant 3$ is $z^{n}$, which implies $H_{A}=H_{3}$. If $h \in\{y x, y z, z x, z z\}, A$ is monomial and therefore the defining relations form a Gröbner basis of $I$. If $h=z z$, the only normal word of degree $\geqslant 3$ is $y z x$ and $H_{A}=H_{2}$. If $h=z x$, the only normal words of degree $n \geqslant 3$ are $z^{n}$ and $y z^{n-1}$, which gives $H_{A}=H_{7}$. If $h=y z$, the only normal words of degree $n \geqslant 3$ are $z^{n-1} x$ and $z^{n}$, yielding $H_{A}=H_{7}$. If $h=y x$, the only normal words of degree $n \geqslant 3$ are $y z^{n-2} x y z^{n-1}, z^{n-1} x$ and $z^{n}$. Hence $H_{A}=H_{9}$.

Finally, assume that (2.5) is satisfied. That is, $A$ is given by the relations $x x, x y, x z, y y, z z$ and $h$ with $h \in\{y x+z x, y z+z y, y x+z x-y z-z y\}$. If $h=y x+z x-y z-z y$, the defining relations together with $y z x, y z y$ and $z y z$ form a Gröbner basis of $I$. There are no normal words of degree $\geqslant 3$ and therefore $H_{A}=H_{1}$. If $h=y z+z y$, the defining relations form a Gröbner basis of $I$. The only 
normal word of degree $\geqslant 3$ is $z y x$ and $H_{A}=H_{2}$. Finally, if $h=y x+z x$, the defining relations together with $y z x$ form a Gröbner basis of $I$. For $n \geqslant 3$ there are exactly 2 normal words of degree $n$ being the monomials in $y$ and $z$ in which $y$ and $z$ alternate: $y z y z \ldots$ and $z y z y \ldots$ Hence $H_{A}=H_{7}$. Since we have exhausted all the options, the proof is complete.

Proof of Lemma 2.4. The fact that $R$ is 6-dimensional is straightforward. Indeed, $R$ is the image of the 6-dimensional space of the symmetric elements of $V \otimes V$ under the invertible linear map $I \otimes T$. Now, replacing the ground field by a field extension does not change the Hilbert series of an algebra given by generators and relations. Hence, without loss of generality we can assume that $\mathbb{K}$ is algebraically closed. This allows us to pick a basis $x_{1}, x_{2}, x_{3}$ in $V$ with respect to which the matrix of $T$ has the Jordan normal form.

If $T$ has 3 Jordan blocks, $T$ has the diagonal matrix with respect to the basis $x_{1}, x_{2}, x_{3}$ with the non-zero numbers (eigenvalues) $\lambda_{1}, \lambda_{2}$ and $\lambda_{3}$ on the diagonal. One easily sees that in this case $R$ is spanned by $x_{j}^{2}$ with $1 \leqslant j \leqslant 3$ and $\lambda_{k} x_{j} x_{k}+\lambda_{j} x_{k} x_{j}$ with $1 \leqslant j<k \leqslant 3$. If $T$ has 2 Jordan blocks, we can assume that the size two block is in the left upper corner and corresponds to the eigenvalue $\lambda$, while the size one block is in the right lower corner and corresponds to the eigenvalue $\mu$. In this case $x_{1}^{2}, \lambda x_{2}^{2}+x_{2} x_{1}, x_{3}^{2}, x_{1} x_{2}+x_{2} x_{1}, \mu x_{1} x_{3}+\lambda x_{3} x_{1}$ and $\mu x_{2} x_{3}+x_{3} x_{1}+\lambda x_{3} x_{2}$ forms a linear basis in $R$. Finally, if $T$ has just one Jordan block corresponding to the eigenvalue $\lambda$, a linear basis in $R$ is formed by $x_{1}^{2}, \lambda x_{2}^{2}+x_{2} x_{1}, \lambda x_{3} x_{1}+\lambda x_{3} x_{2}+x_{3}^{2}, x_{1} x_{2}+x_{2} x_{1}, x_{1} x_{3}+x_{3} x_{1}-x_{2} x_{1}$ and $x_{2} x_{3}+x_{3} x_{2}+x_{3} x_{1}$. In any case, this linear basis in $R$ is also a Gröbner basis in $I(V, R)$ with the only normal word of degree $\geqslant 3$ being $x_{3} x_{2} x_{1}$. This gives $H_{A}=H_{2}$.

This completes the proof of Proposition 2.1. Note that if $\mathbb{K}$ is algebraically closed and $A$ is a quadratic algebra satisfying $H_{A}=H_{j}$ for $j \in\{8,9,10,11\}$, Lemma 1.4 can be applied and $A$ falls into one of the cases considered in the proofs of Lemmas 2.2 and 2.3. Scanning the proofs, one sees that whenever $H_{A}=H_{j}$ for $j \in\{8,9,10,11\}, A$ is actually PBW and therefore Koszul. Since the Hilbert series or Koszulity do not notice an extension of the ground field, we can drop the condition that $\mathbb{K}$ is algebraically closed. This observation automatically implies the following Koszulity result.

Proposition 2.5. If $A$ is a quadratic algebra satisfying $H_{A}=H_{j}$ for $j \in\{8,9,10,11\}$, then $A$ is Koszul. Moreover, $A$ is PBW provided $\mathbb{K}$ is algebraically closed.

\section{Proof of Lemma 1.4}

We start by reformulating Lemma 1.4. First, if we take a pairing on $V \otimes V$ as in the definition of a dual algebra, then in terms of $S=R^{\perp}$, Lemma 1.4 reads in the following way.

Lemma 3.1. Let $V$ be a 3-dimensional vector space over an infinite field $\mathbb{K}$ and $S$ be a 3-dimensional subspace of $V \otimes V$. Then at least one of the following statements is true:

$\left(\mathrm{P} 1^{\prime}\right)$ there is a 2-dimensional subspace $M \subset V$ such that $(V \otimes M) \oplus S=V \otimes V$ or $(M \otimes V) \oplus S=V \otimes V$;

$\left(\mathrm{P}^{\prime}\right)$ there is a 2-dimensional subspace $M \subset V$ such that $V \otimes M \supset S$ or $M \otimes V \supset S$;

$\left(\mathrm{P} 3^{\prime}\right)$ there is an invertible linear map $T: V \rightarrow V$ such that $S=\operatorname{span}\{x \otimes T y-y \otimes T x: x, y \in V\}$.

For two vector spaces $V_{1}$ and $V_{2}$ over $\mathbb{K}, L\left(V_{1}, V_{2}\right)$ stands for the vector space of all linear maps from $V_{1}$ to $V_{2}$. Using the natural isomorphism between $V \otimes V$ and $L\left(V^{*}, V\right)$ together with the fact that a two-dimensional subspace of $V$ is exactly the kernel of a non-zero linear functional, we can reformulate Lemma 3.1 in the following way.

Lemma 3.2. Let $V$ be a 3-dimensional vector space over an infinite field $\mathbb{K}$ and $S$ be a 3-dimensional subspace of $L\left(V^{*}, V\right)$. Then at least one of the following statements is true:

$\left(\mathrm{P} 1^{\prime \prime}\right)$ there is $f \in V^{*}$ such that the map $A \mapsto A f$ or the map $A \mapsto A^{*} f$ from $S$ to $V$ is injective;

$\left(\mathrm{P}^{\prime \prime}\right)$ there is a non-zero $f \in V^{*}$ such that $A f=0$ for all $A \in S$ or $A^{*} f=0$ for all $A \in S$;

$\left(\mathrm{P} 3^{\prime \prime}\right)$ there is an invertible $T \in L(V, V)$ such that $g(T A f)=-f(T A g)$ for all $f, g \in V^{*}$ and $A \in S$. 
In other words, we have to show that $\left(\mathrm{P}^{\prime \prime}\right)$ holds if both $\left(\mathrm{P} 1^{\prime \prime}\right)$ and $\left(\mathrm{P} 2^{\prime \prime}\right)$ fail. Hence, Lemma 3.2 and therefore Lemma 1.4 will follow if we prove the following result.

Lemma 3.3. Let $V_{1}$ and $V_{2}$ be a 3-dimensional vector spaces over an infinite field $\mathbb{K}$ and $S$ be a 3-dimensional subspace of $L\left(V_{1}, V_{2}\right)$. Assume also that

$$
\bigcap_{A \in S} \operatorname{ker} A=\bigcap_{A \in S} \operatorname{ker} A^{*}=\{0\}
$$

(L2) $\{A u: A \in S\} \neq V_{2}$ for each $u \in V_{1}$ and $\left\{A^{*} f: A \in S\right\} \neq V_{1}^{*}$ for each $f \in V_{2}^{*}$.

Then there exist linear bases in $V_{1}$ and $V_{2}$ such that $S$ in the corresponding matrix form is exactly the space of $3 \times 3$ antisymmetric matrices.

Proof. First, we shall show that

$$
\text { for every non-zero } x \in V_{1} \text {, the space } S x=\{A x: A \in S\} \text { is two-dimensional. }
$$

By (L1), $S x \neq\{0\}$ for each $x \in V_{1} \backslash\{0\}$. By (L2), $S x \neq V_{2}$ for each $x \in V_{1}$. Thus $S x$ for $x \in V_{1} \backslash\{0\}$ is either one-dimensional or two-dimensional. Assume that (3.1) fails. Then there is $x_{1} \in V_{1}$ such that $S x_{1}$ is one-dimensional. Then we can pick a basis $A_{1}, A_{2}, A_{3}$ in $S$ such that $A_{1} x_{1}=y_{1} \neq 0$ and $A_{2} x_{1}=A_{3} x_{1}=0$. By (L1), the linear span of the images of all $A \in S$ is $V_{2}$. Hence we can pick $x_{2}, x_{3} \in V_{1}$ such that $x_{1}, x_{2}, x_{3}$ is a basis in $V_{1}$, while $y_{1}, y_{2}, y_{3}$ is a basis in $V_{2}$, where $y_{j}=A_{j} x_{j}$. With respect to the bases $x_{1}, x_{2}, x_{3}$ and $y_{1}, y_{2}, y_{3}$, the matrices of $A_{1}, A_{2}$ and $A_{3}$ have the shape

$$
\left(\begin{array}{lll}
1 & * & * \\
0 & * & * \\
0 & * & *
\end{array}\right),\left(\begin{array}{lll}
0 & 0 & * \\
0 & 1 & * \\
0 & 0 & *
\end{array}\right) \text { and }\left(\begin{array}{lll}
0 & * & 0 \\
0 & * & 0 \\
0 & * & 1
\end{array}\right) \text {, respectively. }
$$

Keeping the basis in $V_{2}$ as well as $x_{1}$ and replacing $x_{2}$ and $x_{3}$ by $x_{2}+\alpha x_{1}$ and $x_{3}+\beta x_{1}$ respectively with appropriate $\alpha, \beta \in \mathbb{K}$, we can kill the second and the third entries in the first row of the first matrix. With respect to the new basis, the matrices of $A_{1}, A_{2}$ and $A_{3}$ are

$$
\left(\begin{array}{ccc}
1 & 0 & 0 \\
0 & a_{1} & a_{2} \\
0 & a_{3} & a_{4}
\end{array}\right),\left(\begin{array}{ccc}
0 & 0 & a_{5} \\
0 & 1 & a_{6} \\
0 & 0 & a_{7}
\end{array}\right) \text { and }\left(\begin{array}{ccc}
0 & a_{8} & 0 \\
0 & a_{9} & 0 \\
0 & a_{10} & 1
\end{array}\right) \text { with } a_{j} \in \mathbb{K} \text {. }
$$

By (L2), for every $u=(x, y, z) \in \mathbb{K}^{3}, A_{1} u, A_{2} u, A_{3} u$ are linearly dependent and $A_{1}^{T} u, A_{2}^{T} u, A_{3}^{T} u$ are linearly dependent as well (here $A_{j}$ stand for the matrices of the linear maps $A_{j}$ ), where $T$ denotes the transpose matrix. Computing these vectors, we see that these conditions read

$$
\operatorname{det}\left(\begin{array}{ccc}
x & a_{1} y+a_{2} z & a_{3} y+a_{4} z \\
a_{5} z & y+a_{6} z & a_{7} z \\
a_{8} y & a_{9} y & a_{10} y+z
\end{array}\right)=\operatorname{det}\left(\begin{array}{ccc}
x & a_{1} y+a_{3} z & a_{2} y+a_{4} z \\
0 & y & a_{5} x+a_{6} y+a_{7} z \\
0 & a_{8} x+a_{9} y+a_{10} z & z
\end{array}\right)=0
$$

for all $x, y, z \in \mathbb{K}$. Since $\mathbb{K}$ is infinite, the above two determinants must be zero as polynomials in $x, y, z$. The first determinant has the shape $x\left(a_{10} y^{2}+a_{6} z^{2}+\left(1+a_{6} a_{10}-a_{7} a_{9}\right) y z\right)+g$ with $g \in \mathbb{K}[y, z]$. Hence $a_{10}=a_{6}=0$ and $a_{7} a_{9}=1$. Taking into account that $a_{10}=a_{6}=0$, we see that the $x y z$-coefficient of the second determinant is $a_{7} a_{9}$. Hence $a_{7} a_{9}=0$, which contradicts $a_{7} a_{9}=1$. This contradiction proves (3.1).

Now we pick a non-zero $u \in V_{1}$. By (L2), there is a non-zero $A_{1} \in S$ such that $A_{1} u=0$. Since $A_{1} \neq 0$, there is $x \in V_{1}$ such that $A_{1} x \neq 0$. Since a Zarisski generic $x$ will do, we can assure the extra condition $S u \neq S x$ (otherwise (L1) is violated). Obviously, $u$ and $x$ are linearly independent. By (3.1), we can find $A_{2} \in S$ such that $A_{1} x$ and $A_{2} x$ are linearly independent. Again suppose $A_{2}$ is Zarisski generic and therefore we can achieve the extra condition that $A_{1} x, A_{2} x$ and $A_{2} u$ are linearly independent (otherwise $S u=S x$ ). Now $y_{1}=-A_{2} u, y_{2}=A_{1} x$ and $y_{3}=A_{2} x$ form a basis in $V_{2}$. By (L2), there is a non-zero $A_{3} \in S$ such that $A_{3} x=0$. Clearly, $A_{j}$ are linearly independent (=they form 
a basis in $S$ ). Pick a basis $x_{1}, x_{2}, x_{3}$ in $V_{1}$ such that $x_{1}=x$ and $x_{3}=u$. With respect to the bases $x_{1}, x_{2}, x_{3}$ and $y_{1}, y_{2}, y_{3}$, the matrices of $A_{1}, A_{2}$ and $A_{3}$ have the form

$$
\left(\begin{array}{lll}
0 & * & 0 \\
1 & * & 0 \\
0 & * & 0
\end{array}\right),\left(\begin{array}{ccc}
0 & * & -1 \\
0 & * & 0 \\
1 & * & 0
\end{array}\right) \text { and }\left(\begin{array}{ccc}
0 & * & * \\
0 & * & * \\
0 & * & *
\end{array}\right) \text {, respectively. }
$$

Keeping the basis in $V_{2}$ as well as $x_{1}$ and $x_{3}$ and replacing $x_{2}$ by $x_{2}+\alpha x_{1}+\beta x_{3}$ with appropriate $\alpha, \beta \in \mathbb{K}$, we can kill the middle entry in the first matrix and the second entry of the first row of the second matrix. With respect to the new basis, the matrices of $A_{1}, A_{2}$ and $A_{3}$ have shape:

$$
\left(\begin{array}{ccc}
0 & a_{1} & 0 \\
1 & 0 & 0 \\
0 & a_{2} & 0
\end{array}\right),\left(\begin{array}{ccc}
0 & 0 & -1 \\
0 & a_{4} & 0 \\
1 & a_{5} & 0
\end{array}\right) \text { and }\left(\begin{array}{ccc}
0 & a_{6} & a_{9} \\
0 & a_{7} & a_{10} \\
0 & a_{8} & a_{11}
\end{array}\right) \quad \text { with } a_{j} \in \mathbb{K}
$$

By (L2), for every vector $u=(x, y, z) \in \mathbb{K}^{3}, A_{1} u, A_{2} u, A_{3} u$ are linearly dependent and $A_{1}^{T} u, A_{2}^{T} u, A_{3}^{T} u$ are linearly dependent. Computing these vectors, we see that these conditions mean:

$$
\operatorname{det}\left(\begin{array}{ccc}
a_{1} y & x & a_{2} y \\
-z & a_{4} y & x+a_{5} y \\
a_{6} y+a_{9} z & a_{7} y+a_{10} z & a_{8} y+a_{11} z
\end{array}\right)=\operatorname{det}\left(\begin{array}{ccc}
y & a_{1} x+a_{2} z & 0 \\
-z & a_{4} y+a_{5} z & x \\
0 & a_{6} x+a_{7} y+a_{8} z & a_{9} x+a_{10} y+a_{11} z
\end{array}\right)=0
$$

for all $x, y, z \in \mathbb{K}$. Since $\mathbb{K}$ is infinite, these two determinants are zero as polynomials in $x, y, z$. The terms containing $x^{2}$ in the first determinant amount to $x^{2}\left(a_{6} y+a_{9} z\right)$, which implies $a_{6}=a_{9}=0$. The $y z^{2}$-coefficient of the same polynomial is $-a_{2} a_{10}$. Hence $a_{2} a_{10}=0$. First, we show that $a_{2}=0$. Indeed, assume the contrary. Then $a_{2} \neq 0$ and therefore $a_{10}=0$. Now $z^{3}$-coefficient in the second determinant is $-a_{2} a_{11}$. Hence $a_{11}=0$. Taking into account that $a_{6}=a_{9}=a_{10}=a_{11}=0$, we see that in the first determinant the terms containing $z$ amount to $z\left(a_{8} x y-a_{2} a_{7} y^{2}\right)$. It follows that $a_{7}=a_{8}=0$ and therefore $A_{3}=0$, which is a contradiction. Hence $a_{2}=0$. Recall that we already know that $a_{6}=a_{9}=0$. Next, we show that $a_{1} \neq 0$. Indeed, assume the contrary: $a_{1}=0$. Then the first determinant simplifies to $z x\left(a_{8} y+a_{11} z\right)$. Hence $a_{8}=a_{11}=0$. Now the second determinant simplifies to $a_{4} a_{10} y^{3}+a_{5} a_{10} y^{2} z-a_{7} x y^{2}$. Hence $a_{7}=0$ and $a_{4} a_{10}=a_{5} a_{10}=0$. If $a_{10}=0$, we have $A_{3}=0$, which is a contradiction. If $a_{10} \neq 0$, we have $a_{4}=a_{5}=0$. In this case the second column of each $A_{j}$ is zero. Thus the second basic vector in $V_{1}$ is in the common kernel of all elements of $S$, which contradicts (L1). These contradictions prove that $a_{1} \neq 0$. By normalizing the second basic vector in $V_{1}$ appropriately, we can assume that $a_{1}=-1$. Taking this into account together with $a_{2}=a_{6}=a_{9}=0$, we see that the $x y^{2}$ and $x z^{2}$ coefficients in the first determinant are $-a_{7}$ and $a_{11}$ respectively. Hence $a_{7}=a_{11}=0$. Now the determinants in the above display simplify to

$$
-a_{4} a_{8} y^{3}+\left(a_{8}+a_{10}\right) x y z+a_{5} a_{10} y^{2} z \text { and } a_{4} a_{10} y^{3}+a_{5} a_{10} y^{2} z-\left(a_{8}+a_{10}\right) x y z .
$$

Since they vanish, $a_{10}=-a_{8}$. If $a_{8}=0$, then $a_{10}=0$ and $A_{3}=0$, which is a contradiction. Thus $a_{8} \neq 0$. Now vanishing of the polynomials in the above display implies $a_{4}=a_{5}=0$. Hence, the matrices $A_{1}, A_{2}$ and $A_{3}$ acquire the shape

$$
\left(\begin{array}{ccc}
0 & -1 & 0 \\
1 & 0 & 0 \\
0 & 0 & 0
\end{array}\right),\left(\begin{array}{ccc}
0 & 0 & -1 \\
0 & 0 & 0 \\
1 & 0 & 0
\end{array}\right) \text { and } a_{8}\left(\begin{array}{ccc}
0 & 0 & 0 \\
0 & 0 & -1 \\
0 & 1 & 0
\end{array}\right) \text { with } a_{8} \neq 0
$$

and $S$ becomes the space of all antisymmetric matrices.

Since Lemma 3.3 is equivalent to Lemma 1.4, the proof of Lemma 1.4 is now complete. 
Table 1: Algebras $A_{j}$ for $1 \leqslant j \leqslant 11$

\begin{tabular}{|l|l|l|l|l|}
\hline$j$ & defining relations of $A_{j}$ & $\begin{array}{l}\text { other elements of } \\
\text { the Gröbner basis }\end{array}$ & $\begin{array}{l}\text { normal words } \\
\text { of degrees } \geqslant 3\end{array}$ & $\begin{array}{l}\text { Hilbert } \\
\text { series }\end{array}$ \\
\hline 1 & $x x-z x, x y-z z, x z, y x, y y-z y, y z$ & $z z x, z z y, z z z$ & none & $H_{1}$ \\
\hline 2 & $x x, y x, y y, z x, z y, z z$ & none & $x y z$ & $H_{2}$ \\
\hline 3 & $x x-z x, x y, x z, y x, y y-z y, y z$ & $z z x, z z y$ & $z^{n}$ for $n \geqslant 3$ & $H_{3}$ \\
\hline 4 & $x x, x y, x z-z z, y x, y y, y z-z z$ & $z z z$ & $z z x, z z y$ & $H_{4}$ \\
\hline 5 & $x x-y x, x y, y y, y z, z x, z z$ & $z y x$ & $x z y, y x z, y x z y$ & $H_{5}$ \\
\hline 6 & $x z-y z, x y, y x, y y, z x, z z$ & $z y z$ & $y z y, x^{n}$ for $n \geqslant 3$ & $H_{6}$ \\
\hline 7 & $x x, x y, x z, y y, z x, z y$ & none & $z^{n}, y z^{n-1}$ for $n \geqslant 3$ & $H_{7}$ \\
\hline 8 & $x y, x z, y x, y z, z x, z y$ & none & $x^{n}, y^{n}, z^{n}$ for $n \geqslant 3$ & $H_{8}$ \\
\hline 9 & $x x, x z, y x, z x, z y, z z$ & none & $y^{n-1}, x y^{n-2} z, y^{n}$, & $H_{9}$ \\
& & & $\begin{array}{l}z^{m} y^{n-m} \text { for } n \geqslant 3 \\
0 \leqslant m \leqslant n\end{array}$ & $H_{10}$ \\
\hline 10 & $x x, x y, x z, y x, y z, z x$ & none & $\begin{array}{l}\text { all monomials in } y, z \\
\text { without } y y \text { as a subword }\end{array}$ & $H_{11}$ \\
\hline 11 & $x x, x y, x z, y x, y y, z x$ & none & & \\
& & & &
\end{tabular}

\section{$4 \quad$ Specific algebras satisfying $H_{A}=H_{j}$}

For each $j \in\{1, \ldots, 11\}$, we provide a quadratic algebra $A_{j}$ (generated by degree 1 elements $x, y$ and $z$ ) satisfying $H_{A_{j}}=H_{j}$. In each case the last equality is an easy exercise since we supply the finite Gröbner basis in the ideal of relations and describe the normal words. These 11 examples are presented in Table 1.

Counting normal words is trivial for all $A_{j}$ except the last one, where the normal words of degree $n \geqslant 3$ are exactly monomials in $z$ and $y$, which do not contain $y y$ as a submonomial. In this case, as it was already observed in the proof of Lemma 2.3, the numbers $a_{n}$ of such monomials of degree $n$ are consecutive Fibonacci numbers with $a_{3}=5$, yielding $H_{A_{11}}=H_{11}$.

Proof of Theorem 1.1. By Proposition 2.1, $H_{A} \in\left\{H_{1}, \ldots, H_{11}\right\}$ for every quadratic $\mathbb{K}$-algebra $A$ satisfying $\operatorname{dim} A_{1}=\operatorname{dim} A_{2}=3$. The examples in Table 1 show that each $H_{j}$ with $1 \leqslant j \leqslant 11$ is indeed the Hilbert series of a quadratic $\mathbb{K}$-algebra.

It remains to deal with Koszulity. We need the following elementary observation.

Lemma 4.1. Assume that $A$ is a degree graded algebra on generators $x_{1}, \ldots, x_{n}$, that the monomials in $x_{j}$ carry a well-ordering compatible with the multiplication and that $\Lambda$ is the set of the leading monomials of all members of the corresponding Gröbner basis of the ideal of relations of $A$. Let also $1 \leqslant j, k \leqslant n$ be such that $x_{j} \neq 0, x_{k} \neq 0$ and $x_{j} x_{k}=0$ in A. Finally, assume that $\Lambda$ contains no monomial ending with $x_{s} x_{k}$ for $s \neq j$. Then for $u \in A, u x_{k}=0 \Longleftrightarrow u=v x_{j}$ for some $v \in A$.

Proof. Since $x_{j} x_{k}=0$ in $A, u x_{k}=v x_{j} x_{k}=0$ if $u=v x_{j}$ for some $v \in A$.

Assume now that $u \in A$ and $u x_{k}=0$. It remains to show that $u=v x_{j}$ for some $v \in A$. Let $\mathcal{N}$ be the set of all normal words for $A$. That is, $\mathcal{N}$ is the set of monomials containing no member of $\Lambda$ as a submonomial. Since $\mathcal{N}$ is a linear basis in $A$, we can write $u$ as a linear combination of elements of $\mathcal{N}$. We also separate those words in this combination ending with $x_{j}$ from the rest of them: $u=\sum_{\alpha} c_{\alpha} w_{\alpha} x_{j}+\sum_{\beta} d_{\beta} v_{\beta}$, where both sums are finite (an empty sum is supposed to be zero), $w_{\alpha} x_{j}, v_{\beta}$ are pairwise distinct normal words, none of $v_{\beta}$ ends with $x_{j}$ and $c_{\alpha}, d_{\beta} \in \mathbb{K}^{*}$. Then

$$
0=u x_{k}=\sum_{\alpha} c_{\alpha} w_{\alpha} x_{j} x_{k}+\sum_{\beta} d_{\beta} v_{\beta} x_{k}=\sum_{\beta} d_{\beta} v_{\beta} x_{k} \text { in } A,
$$


where the last equality is due to $x_{j} x_{k}=0$. Since $v_{\beta}$ does not end with $x_{j}$ and $\Lambda$ contains neither $x_{j}$ nor $x_{k}$ nor any monomial ending with $x_{s} x_{k}$ with $s \neq j$, we easily see that each $v_{\beta} x_{k}$ is a normal word. Since the set of normal words is linearly independent in $A$, the above display implies that the $\operatorname{sum} \sum_{\beta} d_{\beta} v_{\beta}$ is empty and therefore $u=\sum_{\alpha} c_{\alpha} w_{\alpha} x_{j}=v x_{j}$ with $v=\sum_{\alpha} c_{\alpha} w_{\alpha}$.

Proposition 4.2. The algebras $A_{j}$ with $j \in\{2,5,6,7,8,9,10,11\}$ are Koszul.

Proof. For $j \in\{2,7,8,9,10,11\}, A_{j}$ is a monomial algebra, hence it is Koszul. It remains to verify Koszulity of $A_{5}$ and $A_{6}$. Consider the algebra $B$ given by the generators $x, y, z$ and the relations $x x+y x$, $x z, z y$ and the algebra $C$ given by the generators $x, y, z$ and the relations $x x, x z+y z, z y$. A direct computation shows that $B^{!}=A_{5}$ and $C^{!}=A_{6}$. Hence Koszulity of $A_{5}$ and $A_{6}$ is equivalent to Koszulity of $B$ and $C$ respectively. It remains to prove that $B$ and $C$ are Koszul, which is our objective now.

Consider the following sequences of free graded left $B$-modules and $C$-modules:

$$
\begin{aligned}
& 0 \rightarrow B \stackrel{d_{4}}{\longrightarrow} B^{2} \stackrel{d_{3}}{\longrightarrow} B^{3} \stackrel{d_{2}}{\longrightarrow} B^{3} \stackrel{d_{1}}{\longrightarrow} B \stackrel{d_{0}}{\longrightarrow} \mathbb{K} \rightarrow 0, \\
& \ldots \stackrel{\delta_{5}}{\longrightarrow} C \stackrel{\delta_{5}}{\longrightarrow} C \stackrel{\delta_{5}}{\longrightarrow} C \stackrel{\delta_{4}}{\longrightarrow} C^{2} \stackrel{\delta_{3}}{\longrightarrow} C^{3} \stackrel{\delta_{2}}{\longrightarrow} C^{3} \stackrel{\delta_{1}}{\longrightarrow} C \stackrel{\delta_{0}}{\longrightarrow} \mathbb{K} \rightarrow 0,
\end{aligned}
$$

where $d_{0}$ and $\delta_{0}$ are the augmentation maps,

$$
\begin{aligned}
& d_{1}(u, v, w)=u x+v y+w z, d_{2}(u, v, w)=(u(x+y), v z, w x), d_{3}(u, v)=(0, u x, v(x+y)) \\
& d_{4}(u)=(u(x+y), 0), \delta_{1}(u, v, w)=u x+v y+w z, \delta_{2}(u, v, w)=(u x, v z, w(x+y)) \\
& \delta_{3}(u, v)=(u x, v(x+y), 0), \delta_{4}(u)=(u x, 0) \text { and } \delta_{5}(u)=u x .
\end{aligned}
$$

Using the relations of $B$ and $C$, one easily sees that the composition of any two consecutive arrows in both sequences is indeed zero. By definition of Koszulity, the proof will be complete if we show that these sequences are exact. The exactness of (4.1) and (4.2) boils down to verifying the following statements:

$$
\begin{aligned}
& \text { for } u \in B, u(x+y)=0 \Longleftrightarrow u=0, \\
& \text { for } u \in B, u x=0 \Longleftrightarrow u=v(x+y) \text { for some } v \in B, \\
& \text { for } u \in B, u z=0 \Longleftrightarrow u=v x \text { for some } v \in B, \\
& \text { for } u \in C, u x=0 \Longleftrightarrow u=v x \text { for some } v \in C, \\
& \text { for } u \in C, u(x+y)=0 \Longleftrightarrow u=0, \\
& \text { for } u \in C, u z=0 \Longleftrightarrow u=v(x+y) \text { for some } v \in C .
\end{aligned}
$$

Indeed, the exactness of (4.1) at the leftmost $B$ is equivalent to (4.3), its exactness at $B^{2}$ is equivalent to (4.3) and (4.4) and its exactness at the leftmost $B^{3}$ is equivalent to (4.3), (4.4) and (4.5). The exactness of (4.2) at each $C$ which is to the left of $C^{2}$ is equivalent to (4.6), its exactness at $C^{2}$ is equivalent to (4.6) and (4.7), while its exactness at the leftmost $C^{3}$ is equivalent to (4.6), (4.7) and (4.8). Checking the exactness of both complexes at three terms on the right is a straightforward exercise. Alternatively, one can notice that (4.1) and (4.2) are the Koszul complexes of $B$ and $C$ respectively, and that the Koszul complex happens to be exact at three right terms for every quadratic algebra, see [23] (exactness of the Koszul complex at the two right terms holds for every graded algebra generated in degree 1, while the exactness at the term third from the right holds for all quadratic algebras).

Thus it remains to prove (4.3- 4.8). Observe that the defining relations $x x+y x, x z, z y$ of $B$ together with $x y^{k} x+y^{k+1} x, k \geqslant 1$ form the Gröbner basis of the ideal of relations of $B$, while the defining relations $x x, x z+y z, z y$ of $C$ together with $x y z$ form the Gröbner basis of the ideal of relations of $C$. Now a direct application of Lemma 4.1 justifies (4.5) and (4.6). In order to prove the rest, we perform the following linear substitution. Keeping $x$ and $z$ as they were, we set the new $y$ to be $x+y$ in the old variables. This substitution provides an isomorphism of $B$ and the algebra $A$ given by the generators $x, y, z$ and the relations $x z, y x, z x-z y$. These relations together with $z y z$ form 
the Gröbner basis of the ideal of relations of $A$. The same substitution provides an isomorphism of $C$ and the algebra $D$ given by the generators $x, y, z$ and the relations $x x, y z, z x-z y$. These relations together with zyx form the Gröbner basis of the ideal of relations of $D$.

Now we can rewrite (4.3), (4.4), (4.7) and (4.8) in terms of multiplication in $A$ and $D$. Namely, they are equivalent to

$$
\begin{aligned}
& \text { for } u \in A, u y=0 \Longleftrightarrow u=0, \\
& \text { for } u \in A, u x=0 \Longleftrightarrow u=v y \text { for some } v \in A, \\
& \text { for } u \in D, u y=0 \Longleftrightarrow u=0, \\
& \text { for } u \in D, u z=0 \Longleftrightarrow u=v y \text { for some } v \in D,
\end{aligned}
$$

respectively. Again, Lemma 4.1justifies (4.10) and (4.12). Next, one easily sees that the sets of normal words for both $A$ and $D$ are closed under the multiplication by $y$ on the right. This implies (4.9) and (4.11). Hence (4.3-4.8) hold and therefore $B$ and $C$ are Koszul.

Proposition 4.3. Let $A$ be a quadratic algebra such that $H_{A} \in\left\{H_{1}, H_{3}, H_{4}\right\}$. Then $A$ is non-Koszul.

Proof. Assume the contrary. Then $A$ is Koszul and by (1.1),$H_{A^{!}}(t)=\frac{1}{H_{A}(-t)}$. In particular, all coefficients of the series $\frac{1}{H_{A}(-t)}$ must be non-negative. On the other hand,

$\frac{1}{H_{1}(-t)}=1+3 t+6 t^{3}+9 t^{3}+9 t^{4}-27 t^{6}+\cdots$ and $\frac{1}{H_{3}(-t)}=1+3 t+6 t^{3}+10 t^{3}+14 t^{4}+16 t^{5}+12 t^{6}-4 t^{7}+\cdots$

Hence $H_{1}$ and $H_{3}$ can not be Hilbert series of a Koszul algebra.

It remains to consider the case $H_{A}=H_{4}$. Since replacing the ground field by a field extension does not effect the Hilbert series or Koszulity, we can without loss of generality assume that $\mathbb{K}$ is algebraically closed. By Lemmas 1.4, 2.3 and 2.4, $A=A(V, R)$ with $R$ satisfying condition (P1) of Lemma 1.4. Thus, by passing to the algebra with the opposite multiplication, if necessary, we can assume that $R \oplus(L \otimes V)=V \otimes V$ for a 1-dimensional subspace $L$ of $V$. Now choose a basis $x, y, z$ in $V$ such that $x$ spans $L$. Then $R^{\perp} \oplus(M \otimes V)=V \otimes V$, where $M=\operatorname{span}\{y, z\}$. It follows that we can choose a basis $f, g, h$ in $R^{\perp}$ such that the leading monomials of $f, g$ and $h$ are $x x, x y$ and $x z$ respectively. Since $H_{A}=H_{4}=1+3 t+3 t^{2}+2 t^{3}$, a direct computation shows that

$$
H_{A^{!}}=\frac{1}{H_{A}(-t)}=1+3 t+6 t^{2}+11 t^{3}+21 t^{4}+42 t^{5}+85 t^{6}+\cdots
$$

(we need few first coefficients). Since $\operatorname{dim} A_{3}^{!}=11$, there should be exactly one degree 3 element $q$ of the Gröbner basis of the ideal of relations of $A$ ! . The leading monomial $\bar{q}$ of $q$ can have either the shape $u_{1} u_{2} x$ or the shape $u_{1} u_{2} u_{3}$, where $u_{j} \in\{y, z\}$. First, assume that $\bar{q}=u_{1} u_{2} u_{3}$. Then $A_{4}^{!}$is spanned by $v_{1} v_{2} v_{3} x$ with $v_{j} \in\{y, z\}$ and $v_{1} v_{2} v_{3} \neq u_{1} u_{2} u_{3}$ and by $v_{1} v_{2} v_{3} v_{4}$ with $v_{j} \in\{y, z\}$ and $v_{1} v_{2} v_{3} \neq u_{1} u_{2} u_{3}$, $v_{2} v_{3} v_{4} \neq u_{1} u_{2} u_{3}$. The number of these monomials is 20 if $u_{1}=u_{2}=u_{3}$ and is 19 otherwise. Thus $\operatorname{dim} A_{4}^{!} \leqslant 20$. Since by the above display $\operatorname{dim} A_{4}^{!}=21$, we have arrived to a contradiction, which proves that $\bar{q}$ can not be of the shape $u_{1} u_{2} u_{3}$.

Hence $\bar{q}=u_{1} u_{2} x$ with $u_{1}, u_{2} \in\{y, z\}$. In this case, were the relations $f, g, h$ together with $q$ is the Gröbner basis, the dimension of $A_{4}^{!}$would have been 22 . Since $\operatorname{dim} A_{4}^{!}=21$, there is exactly one degree 4 element $p$ of the Gröbner basis of the ideal of relations of $A^{\text {! }}$. The leading monomial $\bar{p}$ of $p$ can have either the shape $w_{1} w_{2} w_{3} x$ or the shape $w_{1} w_{2} w_{3} w_{4}$, where $w_{j} \in\{y, z\}$. Again, first, assume that $\bar{p}=w_{1} w_{2} w_{3} w_{4}$. Then $A_{5}^{!}$is spanned by $v_{1} v_{2} v_{3} v_{4} x$ with $v_{j} \in\{y, z\}$ and $v_{3} v_{4} \neq u_{1} u_{2}$ and by $v_{1} v_{2} v_{3} v_{4} v_{5}$ with $v_{j} \in\{y, z\}$ and $v_{1} v_{2} v_{3} v_{4} \neq w_{1} w_{2} w_{3} w_{4}, v_{2} v_{3} v_{4} v_{5} \neq w_{1} w_{2} w_{3} w_{4}$. It easily follows that $\operatorname{dim} A_{5}^{!} \leqslant 41$. Since by the above display $\operatorname{dim} A_{5}^{!}=42$, we have arrived to a contradiction, which proves that $\bar{p}$ can not be of the shape $w_{1} w_{2} w_{3} w_{4}$.

Hence $\bar{p}=w_{1} w_{2} w_{3} x$ with $w_{j} \in\{y, z\}$ and $w_{2} w_{3} \neq u_{1} u_{2}$. In this case, $A_{6}^{!}$is spanned by 64 elements $v_{1} v_{2} v_{3} v_{4} v_{5} v_{6}$ with $v_{j} \in\{y, z\}$ and by 20 elements $v_{1} v_{2} v_{3} v_{4} v_{5} x$ with $v_{j} \in\{y, z\}, v_{4} v_{5} \neq u_{1} u_{2}$, $v_{3} v_{4} v_{5} \neq w_{1} w_{2} w_{3}$. Hence $\operatorname{dim} A_{6}^{!} \leqslant 84$. Since by the above display $\operatorname{dim} A_{6}^{!}=85$, we have arrived to a contradiction. Thus $H_{4}$ is not the Hilbert series of a Koszul algebra. 
Proof of Theorem 1.2. By Proposition 4.2, for $j \in\{2,5,6,7,8,9,10,11\}$, there is a Koszul algebra $A$ satisfying $H_{A}=H_{j}$. By Proposition 4.3, every quadratic algebra $A$ satisfying $H_{A}=H_{j}$ with $j \in\{1,3,4\}$ is non-Koszul.

\subsection{Some remarks}

1. The condition of $\mathbb{K}$ being infinite in Lemma 1.4 can be relaxed to $\mathbb{K}$ having sufficiently many elements. More precisely, examining closely the idea behind the proof, one gets that Lemma 1.4 holds if the condition of $\mathbb{K}$ being infinite is relaxed to $|\mathbb{K}| \geqslant 4$. On the other hand, the following example shows that the conclusion of Lemma 1.4 fails if $|\mathbb{K}|=2$.

Example 4.4. Let $x, y, z$ be a basis of a 3 -dimensional vector space $V$ over the 2 -element field $\mathbb{K}=\mathbb{Z}_{2}$. Let also $R \subset V \otimes V$ be the linear span of $x \otimes x, y \otimes y, z \otimes z, y \otimes z+z \otimes y, x \otimes y+z \otimes x+z \otimes y$ and $x \otimes z+y \otimes x+z \otimes y$. Then $R$ is a 6-dimensional subspace of $V \otimes V$ for which each of the conditions (P1-P3) of Lemma 1.4 fails.

We leave the verification to the reader. It can be done by brute force since $V^{+}=V \backslash\{0\}$ has just 7 elements. For example, to show that (P1) fails, one has to find for every $u \in V^{+}, v, w \in V^{+}$such that $u \otimes v, w \otimes u \in R$. Note that extending $\mathbb{K}$ to a 4 -element field forces $R$ from the above example to satisfy (P1). We do not know whether the conclusion of Lemma 1.4 holds if $|\mathbb{K}|=3$.

2. By Proposition 2.5, $A$ is automatically Koszul if $H_{A}=H_{j}$ for $j \in\{8,9,10,11\}$. Note that if $A$ is a quadratic algebra satisfying $H_{A}=H_{j}$ with $j \in\{2,5,6,7\}$, this does not necessarily mean that $A$ is Koszul. We construct the following examples to illustrate this. In these examples we assume $|\mathbb{K}|>2$ and $\alpha \in \mathbb{K}$ is an arbitrary element different from 0 or 1 . Table 2 is completed by computing the Gröbner bases of ideals of relations of algebras $B_{j}$.

Table 2: Algebras $B_{j}$ for $j \in\{2,5,6,7\}$

\begin{tabular}{|l|l|l|l|l|}
\hline$j$ & relations of $B_{j}$ & $\begin{array}{l}\text { other elements of } \\
\text { the Gröbner basis }\end{array}$ & $H_{B_{j}}$ & relations of $B_{j}^{!}$ \\
\hline 2 & $x x+y z, x z, y x, y y+z x, z y, z z$ & $y z x-z x y, x y z-z x y$ & $H_{2}$ & $x x-y z, x y, y y-z x$ \\
\hline 5 & $\begin{array}{l}x x-z x, x y-z x, y x-z x, y y-z x, \\
x z+\alpha z x-\frac{1}{1-\alpha} z z, y z+\alpha z x-\frac{1}{1-\alpha} z z\end{array}$ & $z z x, z z z z$ & $H_{5}$ & $\begin{array}{l}z y, x z+y z+(1-\alpha) z z, \\
x x+x y+y x+y y+z x+\alpha(1-\alpha) z z\end{array}$ \\
\hline 6 & $x x-\alpha z x, x y-z y, y x, x z-\alpha z x, y z, y y$ & $z z x, z z z y$ & $H_{6}$ & $x x+x z+\frac{1}{\alpha} z x, x y+z y, z z$ \\
\hline 7 & $x x-z x, x y, y x, x z-\alpha z x, y z, y y$ & $z z x$ & $H_{7}$ & $x x+\alpha x z+z x, z y, z z$ \\
\hline
\end{tabular}

Computing the Gröbner bases of ideals of relations of algebras $B_{j}^{!}$up to degree 4 , we easily obtain the data presented in Table 3.

Table 3: The series $\left(H_{B_{j}}(-t)\right)^{-1}$ and $H_{B_{j}^{!}}(t)$ up to degree 4

\begin{tabular}{|l|l|l|}
\hline$j$ & $\left(H_{B_{j}}(-t)\right)^{-1}$ up to $t^{4}$ & $H_{B_{j}^{!}}(t)$ up to $t^{4}$ \\
\hline 2 & $1+3 t+6 t^{2}+10 t^{3}+15 t^{4}+\cdots$ & $1+3 t+6 t^{2}+10 t^{3}+17 t^{4}+\cdots$ \\
\hline 5 & $1+3 t+6 t^{2}+11 t^{3}+20 t^{4}+\cdots$ & $1+3 t+6 t^{2}+11 t^{3}+21 t^{4}+\cdots$ \\
\hline 6 & $1+3 t+6 t^{2}+11 t^{3}+20 t^{4}+\cdots$ & $1+3 t+6 t^{2}+11 t^{3}+21 t^{4}+\cdots$ \\
\hline 7 & $1+3 t+6 t^{2}+11 t^{3}+19 t^{4}+\cdots$ & $1+3 t+6 t^{2}+11 t^{3}+20 t^{4}+\cdots$ \\
\hline
\end{tabular}

Table 3 ensures that each $B_{j}$ fails to satisfy $H_{B_{j}}(-t) H_{B_{j}^{!}}(t)=1$ and therefore each $B_{j}$ is non-Koszul. Thus the following statement holds true.

Proposition 4.5. Assuming $|\mathbb{K}|>2$, for each $j \in\{2,5,6,7\}$, there is a non-Koszul quadratic algebra $B$ satisfying $H_{B}=H_{j}$. 
3. By the duality formula (1.1), Theorem 1.2 implies the list of all Hilbert series of Koszul algebras $A$ satisfying $\operatorname{dim} A_{1}=3$ and $\operatorname{dim} A_{2}=6$. They are the series $1 / H(-t)$ for $H$ from the list specified in Theorem 1.2. Thus we have the following corollary.

Corollary 4.6. For Koszul algebras $A$ satisfying $\operatorname{dim} A_{1}=3$ and $\operatorname{dim} A_{2}=6$, the complete list of Hilbert series consists of $\frac{1}{H_{j}(-t)}$ with $j \in\{2,5,6,7,8,9,10,11\}$.

4. A quadratic algebra $A$ satisfying $H_{A}(t) H_{A^{!}}(-t)=1$ is called numerically Koszul. There are examples of numerically Koszul quadratic algebras which are not Koszul, see 23. While proving Proposition 4.3, we have actually shown that $H_{j}$ with $j \in\{1,3,4\}$ can not be the Hilbert series of a numerically Koszul quadratic algebra. We do not know an answer to the following question.

Question 4.7. Let $A$ be a numerically Koszul quadratic algebra satisfying $\operatorname{dim} A_{1}=\operatorname{dim} A_{2}=3$. Is it true that $A$ is Koszul?

We are especially interested in the following particular case.

Question 4.8. 1 Let $A$ be a quadratic algebra satisfying $H_{A}(t)=(1-t)^{-3}$ and $H_{A^{!}}(t)=(1+t)^{3}$. Is it true that $A$ is Koszul?

Note that $(1-t)^{-3}$ is the Hilbert series of $\mathbb{K}[x, y, z]$. The following example shows that for a quadratic algebra $A$, the equality $H_{A}=(1-t)^{-3}$ alone does not guarantee numeric Koszulity.

Example 4.9. Let $A$ be the quadratic algebra given by the generators $x, y, z$ and the relations $x x$, $x z+y y+z x, x y+y x+z z$. Then $H_{A}=(1-t)^{-3}$, while $H_{A^{!}}=H_{3}$. In particular, $A$ is not numerically Koszul and therefore is non-Koszul.

Proof. A direct computation shows that the defining relations of $A$ together with $y y z-z y y$ and $y z z-z y y$ form a Gröbner basis for the ideal of relations of $A$. Now one easily sees that the normal words for $A$ are $z^{k}(y z)^{l} y^{m} x^{\varepsilon}$ with $k, l, m \in \mathbb{Z}_{+}$and $\varepsilon \in\{0,1\}$ and that the number of normal words of degree $n$ is $\frac{(n+1)(n+2)}{2}$. Hence $H_{A}(t)=(1-t)^{-3}$. The dual $A^{!}$is given by the relations $y z, z y, y y-z x, x z-z x$, $x y-z z$ and $y x-z z$, which together with $z x x, z z x$ and $z z z$ form a Gröbner basis for the ideal of relations of $A^{!}$. The only normal word of degree $n \geqslant 3$ is $x^{n}$, which gives $H_{A^{!}}(t)=H_{3}$.

5. The following question remains open.

Question 4.10. Which series feature as the Hilbert series of quadratic algebras satisfying $\operatorname{dim} A_{1}=3$ and $\operatorname{dim} A_{2}=4$ ? Which of these occur for Koszul $A$ ?

The answer to the above question would complete the list of Hilbert series of Koszul algebras $A$ satisfying $\operatorname{dim} A_{1}=3$. In $[23$, it is mentioned that it is unknown whether there is a Koszul algebra $A$ satisfying $\operatorname{dim} A_{1}=3, \operatorname{dim} A_{2}=4$ and $\operatorname{dim} A_{3}=3$. It is important to answer also because if such an algebra exists, it would provide a counterexample to the conjecture on rationality of the Hilbert series of Koszul modules over Koszul algebras. However if such an algebra does not exist, nothing can be derived about rationality.

\section{Acknowledgements:}

We are grateful to IHES and MPIM for hospitality, support, and excellent research atmosphere. We would like to thank anonymous referees for careful reading and useful comments. This work is funded by the ERC grant 320974, and partially supported by the project PUT9038.

\footnotetext{
${ }^{1}$ In the meantime we have acquired an affirmative solution of this question
} 


\section{References}

[1] D. Anick, Generic algebras and $C W$ complexes, Algebraic topology and algebraic $K$-theory (Princeton, N.J., 1983), 247-321, Ann. of Math. Stud. 113, Princeton Univ. Press, Princeton, NJ, 1987

[2] D. Anick, Noncommutative graded algebras and their Hilbert series, J. Algebra 78 (1982), 120-140

[3] M. Artin and W. Shelter, Graded algebras of global dimension 3, Adv. in Math. 66 (1987), 171-216.

[4] Artin, M.; Tate, J.; Van den Bergh, M. Modules over regular algebras of dimension 3 Invent.Math. 106 (1991), 335-388.

[5] Artin, M.; Tate, J.; Van den Bergh, M. Some algebras associated to automorphisms of elliptic curves. The Grothendieck Festschrift, Vol. I, 33 - 85, Progr. Math., 86, Birkhäuser Boston, Boston, MA, 1990.

[6] Bocklandt, Raf; Schedler, Travis; Wemyss, Michael Superpotentials and higher order derivations. J. Pure Appl. Algebra 214 (2010), no. 9, 1501-1522.

[7] P. Cameron and N. Iyudu, Graphs of relations and Hilbert series, J. Symbolic Comput. 42 (2007), 1066-1078

[8] Alain Connes, Michel Dubois-Violette, Non commutative finite dimensional manifolds II. Moduli space and structure of non commutative 3-spheres, Communications in Mathematical Physics 281 (2008), 23-127

[9] V. Drinfeld, On quadratic quasi-commutational relations in quasi-classical limit, Selecta Math. Sovietica 11 (1992), 317-326.

[10] Dubois-Violette, Michel Graded algebras and multilinear forms. C. R. Math. Acad. Sci. Paris 341 2005, no. 12, 719-724.

[11] Dubois-Violette, Michel Multilinear forms and graded algebras. J. Algebra 3172007, no. 1, 198-225.

[12] Ershov, M., Golod - Shafarevich groups: A survey. Int. J. Algebra Comput. 22(2012), N5, 1-68

[13] E. Golod and I. Shafarevich, On the class field tower (Russian), Izv. Akad. Nauk SSSR Ser. Mat. 28 (1964), 261-272.

[14] N. Iyudu and S. Shkarin, The Golod-Shafarevich inequality for Hilbert series of quadratic algebras and the Anick conjecture, Proc. Roy. Soc. Edinburgh A141 (2011), 609-629

[15] N. Iyudu and S. Shkarin, Finite dimensional semigroup quadratic algebras with minimal number of relations, Monatsh. Math. 168 (2012), 239-252

[16] N. Iyudu and S. Shkarin, Asymptotically optimal k-step nilpotency of quadratic algebras and the Fibonacci numbers, Combinatorica [to appear]

[17] N. Iyudu and S. Shkarin, Optimal 5-step nilpotent quadratic algebras, J. Algebra 412 (2014), 1-14

[18] Kontsevich, Maxim, Formal (non)commutative symplectic geometry. The Gel'fand Mathematical Seminars, 1990-1992, 173-187, Birkhuser Boston, Boston, MA, 1993.

[19] Kontsevich, Maxim, Rosenberg, Alexander Noncommutative smooth spaces. arXivmath/9812158

[20] T. H. Lenagan and Agata Smoktunowicz, An infinite dimensional affine nil algebra with finite GelfandKirillov dimension, J. Amer. Math. Soc. 20 (2007), 989-1001.

[21] Manin Yu.I. Some remarks on Koszul algebras and quantum groups Annales de l'institut Fourier 37 (1987) n.4, p.191-205

[22] Odesskii, A. V.; Feigin, B. L. Sklyanin's elliptic algebras. (Russian) Funktsional. Anal. i Prilozhen. 23 (1989), no. 3, 45-54; translation in Funct. Anal. Appl. 23 (1990), no. 3, 207-214

[23] A. Polishchuk and L. Positselski, Quadratic algebras, University Lecture Series 37 American Mathematical Society, Providence, RI, 2005

[24] Priddy, Stewart Koszul resolutions and the Steenrod algebra. Bull. Amer. Math. Soc. 76 (1970), no. 4, 834-839

[25] S.Sierra, D. Rogalski, J. T. Stafford, Noncommutative blowups of elliptic algebras Algebras and Representation Theory, 18 (2015), 491-529.

[26] Sklyanin, E. K. Some algebraic structures connected with the Yang-Baxter equation. Representations of a quantum algebra. (Russian)Funktsional. Anal. i Prilozhen, 17 (1983), no. 4, 34-48.

[27] Zelmanov, E. Some open problems in the theory of infinite dimensional algebras, J. Korean Math. Soc. 44(2007), N5, 1185-1195.

NATAlia IYUdu

SChOOL OF MATHEMATics

The University of Edinburgh

James Clerk Maxwell Building 
The King's Buildings

Peter Guthrie Tait Road

EDINBURGH

SCOTLAND EH9 3FD

E-MAIL ADDRESS: niyudu@staffmail.ed.ac.uk

StanislaV SHKarin

QueEns's University Belfast

Department of Pure Mathematics

UNIVERSITY ROAD, BELFAST, BT7 1NN, UK

E-MAIL ADDRESSES: s.shkarin@qub.ac.uk 